\title{
Weighted gene co-expression network analysis to investigate the key genes implicated in global brain ischemia/reperfusion injury in rats
}

\author{
Dingying Ma ${ }^{1, A, C}$, Jun Qiao ${ }^{2, A, B}$, Qiang $Q u^{3, B, F}$, Fei He ${ }^{4, A, D}$, Wenhua Chen ${ }^{3,4, D-F}, B o Y^{4}{ }^{4, C D, F}$ \\ ${ }^{1}$ Department of Rehabilitation Medicine, Ningbo No. 9 Hospital, China \\ ${ }^{2}$ Department of Rehabilitation, Shanghai Second Rehabilitation Hospital, China \\ ${ }^{3}$ Department of Rehabilitation, School of International Medical Technology, Shanghai Sanda University, China \\ ${ }^{4}$ Department of Rehabilitation Medicine, Shanghai General Hospital, Shanghai Jiaotong University, China \\ A - research concept and design; B - collection and/or assembly of data; $C$ - data analysis and interpretation; \\ $D$ - writing the article; $E$ - critical revision of the article; $F$ - final approval of the article
}

Address for correspondence

Bo Yu

E-mail: Bobby_JTU@163.com

\section{Funding sources}

Shanghai Science and Technology Committee (grant No.16411955200) and Scientific Research and Innovation Team Funding Plan of Shanghai Sanda University, China.

\section{Conflict of interest}

None declared

Received on November 28, 2018

Reviewed on January 3, 2019

Accepted on May 1, 2020

Published online on June 19, 2020

Cite as

Ma D, Qiao J, Qu Q, He F, Chen W, Yu B. Weighted gene co-expression network analysis to investigate the key genes implicated in global brain ischemia/reperfusion injury in rats. Adv Clin Exp Med. 2020;29(6):649-659. doi:10.17219/acem/121918

DOI

10.17219/acem/121918

\section{Copyright}

Copyright by Author(s)

This is an article distributed under the terms of the

Creative Commons Attribution 3.0 Unported (CC BY 3.0)

(https://creativecommons.org/licenses/by/3.0/)

\begin{abstract}
Background. Ischemia/reperfusion (I/R) refers to situations where blood is perfused into ischemic or hypoxic tissues, potentially resulting in an inflammatory response and oxidative injury.

Objectives. This study was conducted to explore the pathogenesis of I/R injury.

Material and methods. GSE82146 was extracted from the Gene Expression Omnibus, consisting of 15 complete global brain ischemia (GGI) reperfusion hippocampus samples and 12 non-ischemic control (NIC) hippocampus samples. The differentially expressed genes (DEGs) between the CGBl and NIC samples were selected using LIMMA package, and were then analyzed with weighted gene co-expression network analysis (WGCNA). Using DAVID software, the DEGs in significant modules were run through enrichment analysis. The DEGs in significant modules were merged, and then a protein-protein interaction (PPI) network was built for them using Cytoscape software. After miRNAs and transcription factors (TFs) were predicted for the DEGs using the WebGestalt tool, a TF-miRNA-target regulatory network was built using Cytoscape software. Furthermore, quantitative real-time polymerase chain reaction (qRT-PCR) analysis was conducted to detect the levels of key genes.
\end{abstract}

Results. There were 390 DEGs in the CGBI samples. Based on WGCNA, brown and turquoise modules were screened as CGBI-associated modules. In the PPI network, key nodes HSP9OAA7 and HSPA5 were able to interact with each other. In the regulatory network, MYC, HSFI and miR-22 had higher degree values. Moreover, HSPA5 was targeted by MYC in the regulatory network. In addition, upregulated HSPBI and HMOX1, as well as downregulated NRAA2, were confirmed with qRT-PCR analysis.

Conclusions. HSPB1, HMOX1 and NRAA2 were the key genes correlated with I/R injury. Additionally, HSP9OAAT, HSPA5, MYC, HSF1, and miR-22 might be related to the pathogenesis of I/R injury.

Key words: ischemia/reperfusion, differentially expressed genes, regulatory network, protein-protein interaction network, weighted gene co-expression network analysis 


\section{Introduction}

Ischemia/reperfusion (I/R) refers to a situation where blood is perfused into tissues experiencing ischemia or hypoxia. ${ }^{1}$ Although I/R promotes the repair of damage and the recovery of functioning in most cases, it can also lead to an inflammatory response and oxidative injury by inducing oxidative stress. ${ }^{2}$ Ischemia/reperfusion is usually related to microvascular injury, and the imbalance of reactive oxygen species (ROS) and nitric oxide (NO) produced by activated endothelial cells is responsible for the subsequent inflammatory response., ${ }^{3,4}$ The development of I/R injury is influenced by ischemia time, aerobic degree, collateral circulation, and reperfusion conditions. The I/R injury has a powerful influence on the ischemic cascade of the brain, involving brain trauma and stroke. ${ }^{5}$ Hence, the molecular mechanisms of I/R injury need to be investigated to better alleviate its adverse effects in clinical practice.

By inhibiting nuclear factor- $k \mathrm{~B}(N F-\kappa B)$, ginkgolide $\mathrm{B}$ $(G B)$ possesses anti-apoptotic and anti-inflammatory effects and has demonstrated neuroprotective roles in mice with ischemia-induced brain injury. ${ }^{6,7}$ The inhibition of P2X7 receptors (P2X7Rs) protects rats from cerebral $\mathrm{I} / \mathrm{R}$ injury by decreasing inflammatory response and may serve as a novel therapeutic approach for transient global cerebral I/R injury. By increasing B-cell leukemia-2 (Bcl-2) expression and reducing $\mathrm{Bcl}-2$-associated $\mathrm{X}$ protein $(\mathrm{Bax})$ expression, propofol functions as a neuroprotective agent in I/R rats. ${ }^{8}$ Oxymatrine can protect the brain of stroke rats from focal I/R injury, and the activation of the nuclear factor erythroid 2-related factor 2 (Nrf2)/hemeoxygenase-1 (HO-1) pathway may promote the neuroprotective effects of oxymatrine in the focal brain I/R rat model. ${ }^{9} \mathrm{MiR}-124$ mediates the expression of $\mathrm{Ku}$ autoantigen $70(\mathrm{Ku} 70)$ and helps to reduce the neuronal death and brain dysfunction caused by I/R. MiR-134 downregulation relieves cerebral ischemic injury through regulating the enhancing cyclic AMP (cAMP) response element-binding protein (CREB) and downstream genes, which provides a potential therapeutic target for the injury. ${ }^{10}$ Nevertheless, the genes and miRNAs affecting brain I/R injury have not been thoroughly explored.

In 2016, Wang et al. performed differential expression analysis on I/R in hippocampus CA1 and CA3, and found that CA3 is better at handling ischemic stress. However, the pathogenesis of I/R injury was not comprehensively researched by them. ${ }^{11}$ To further identify the key genes and miRNAs involved in I/R injury, within this study, a series of bioinformatics analyses was carried out (such as differential expression analysis, weighted gene co-expression network analysis (WGCNA), enrichment analysis, and network analysis) on the expression profile data uploaded by Wang et al. ${ }^{11}$ In addition, quantitative real-time polymerase chain reaction (qRT-PCR) analysis was conducted to confirm the key genes.

\section{Material and methods}

\section{Data source}

The microarray dataset GSE82146 (species: Rattus norvegicus) from the Gene Expression Omnibus database (GEO, http://www.ncbi.nih.gov/geo) was extracted, which was determined on the platform of GPL17117 (RaGene-2_0-st) Affymetrix Rat Gene 2.0 ST Array (transcript (gene) version). There were 15 complete global brain ischemia (CGBI) reperfusion hippocampus samples and 12 non-ischemic control (NIC) hippocampus samples in GSE82146. In Long Evans rats (male, 275-300 g), CGBI was induced with the two-vessel bilateral carotid artery occlusion and hypovolemic hypotension model, ${ }^{12}$ as previously described. ${ }^{13-15}$ Our research was approved by the ethics committee of Ningbo No. 9 Hospital, China.

\section{Differential expression analysis}

The original data in CEL format was downloaded and preprocessed (including format conversion, filling in of the missing data, background correction (MicroArray Suite method), and data standardization (quartile method)) using the R oligo package (v. 1.36.1; http://www.bioconductor.org/packages/release/bioc/html/oligo.html) ${ }^{16}$ Next, the corresponding genes of the probes were annotated based on the annotation platform. For genes with multiple expression values (mapped to multiple probes), the average value was calculated as the unique expression value.

Using the R LIMMA package (v. 3.10.3; http://www.bioconductor.org/packages/2.9/bioc/html/limma.html), ${ }^{17} \mathrm{dif}-$ ferential expression analysis between the CGBI and NIC samples was carried out. The differentially expressed genes (DEGs) were selected using the thresholds of adjusted p-value $<0.05$ and of logfold change $(F C)>0.5$.

\section{WGCNA to identify disease-associated modules and genes}

The WGCNA is a typical algorithm in system biology for constructing a gene co-expression network which can be used to identify modules of the relevant genes. ${ }^{18}$ To screen the CGBI-associated modules and genes, the expression values of the DEGs in each group were determined with WGCNA. ${ }^{18}$ The detailed processes of network building and module identification included consistency analysis between the datasets, the definition of gene co-expressioncorrelated matrix (the correlation coefficient between gene $m$ and gene $n$ was $\left.S_{m n}=\left|\operatorname{cor}_{(m, n)}\right|\right)$, the definition of the adjacent function (the adjacent function was $a_{m n}=\operatorname{power}_{\left(S_{m n, \beta}\right)}$ ), the determination of the parameters for the adjacent function (the weighting coefficient $\beta \geq 0.8$ ), the measurement of the degree of dissimilarity between the nodes, the identification of gene modules (the number of genes in the module $\geq 30$ ), and the determination of the correlation between network module and disease state. 


\section{Enrichment analysis}

Using DAVID software (v. 6.8; https://david-d.ncifcrf. gov/), ${ }^{19}$ Gene Ontology $(\mathrm{GO})^{20}$ and Kyoto Encyclopedia of Genes and Genomes (KEGG), ${ }^{21}$ enrichment analyses were conducted for the DEGs in significant modules. The thresholds for selecting significant terms were p-values $<0.05$ and counts of involved genes $\geq 2$.

\section{Protein-protein interaction (PPI) network analysis}

After the DEGs in significant modules were merged, they were input into the STRING database (v. 10.0; http:// www.string-db.org/ $/)^{22}$ to predict the PPIs among them. The species was rat and the parameter PPI score was set at 0.4. The PPI results were downloaded in TSV format and Cytoscape (v. 3.2.0; http://www.cytoscape.org//23 was used to construct the PPI network.

\section{Transcription factor-miRNA-target regulatory network analysis}

Using the WebGestalt tool (http://www.webgestalt. org/), ${ }^{24}$ miRNAs and transcription factors (TFs) were predicted for the DEGs involved in the PPI network using Overrepresentation Enrichment Analysis (ORA). The species was rat and the reference background was the Affymetrix Rat Gene v. 2.0 ST platform. According to significance levels, the top 10 results of miRNA-target and TF-target were obtained and integrated. Then, a TFmiRNA-target regulatory network was built using Cytoscape software. ${ }^{23}$

\section{qRT-PCR analysis}

The total RNA of 7 brain I/R tissues and 7 control tissues were isolated using a Trizol total RNA extraction kit (Invitrogen, Shanghai, China) following the manufacturer's instructions. The purity and integrity of RNA were evaluated separately by spectrophotometer (Merinton, Beijing, China) and $2 \%$ agarose gel electrophoresis. The primer sequences of key genes were designed for qRT-PCR experiments (Table 1), and were then produced by Sangon Biotech Co., Ltd. (Shanghai, China). The qRT-PCR experiments were carried out using SYBR Green master mix kit (Applied Biosystems, Foster City, USA). The 20- $\mu$ L PCR amplification system consisted of $10 \mu \mathrm{L}$ of SYBR Premix Ex Taq ( $\times 2), 8 \mu \mathrm{L}$ of cDNA template (keeping a consistent level after being diluted with $\left.\mathrm{ddH}_{2} \mathrm{O}\right), 1 \mu \mathrm{L}$ of forward primer $(10 \mu \mathrm{M})$, and $1 \mu \mathrm{L}$ of reverse primer $(10 \mu \mathrm{M})$. The reaction conditions were 40 cycles of $50^{\circ} \mathrm{C}$ for $3 \mathrm{~min}, 95^{\circ} \mathrm{C}$ for $3 \mathrm{~min}$, $95^{\circ} \mathrm{C}$ for $10 \mathrm{~s}$, and $60^{\circ} \mathrm{C}$ for $30 \mathrm{~s}$. Afterwards, a melt curve was created. All experiments were repeated 3 times, and glyceraldehyde-3-phosphate dehydrogenase (GAPDH) was utilized as the reference gene.
Table 1. The primer sequences designed for qRT-PCR experiments

\begin{tabular}{|l|c|}
\multicolumn{1}{|c|}{ Primer names } & Primer sequences $\left(5^{\prime}{ }^{\prime}{ }^{\prime}\right.$ ) \\
\hline GAPDH-rF & AGACAGCCGCATCTTCTTGT \\
\hline GAPDH-rR & CTTGCCGTGGGTAGAGTCAT \\
\hline HSPB1-rF & GATCACTGGCAAGCACGAAG \\
\hline HSPB1-rR & CCTGGAGGGAGCGTGTATTT \\
\hline HMOX1-rF & CGTGCTCGCATGAACACTCT \\
\hline HMOX1-rR & GGCCTCTGGCGAAGAACTC \\
\hline NR4A2-rF & CTGTCAGCATTACGGTTTCG \\
\hline NR4A2-rR & ATCCTGTGGGCTCTTCGGTT \\
\hline
\end{tabular}

\section{Statistical analysis}

The expression levels of the key genes were analyzed using the $2^{-\Delta \Delta \mathrm{Ct}}$ method. ${ }^{25}$ All data are presented as mean \pm standard error of the mean (SEM). SPSS v. 22.0 software (SPSS Inc., Chicago, USA) was used to perform statistical analysis, with $\mathrm{p}<0.05$ serving as the threshold of statistical significance.

\section{Results}

\section{Differential expression analysis}

There were 390 DEGs in the CGBI samples compared with the NIC samples, including 330 upregulated genes (such as heat shock protein B1 (HSPB1) and heme oxygenase (decycling) 1 (HMOX1)), and 60 downregulated genes (such as nuclear receptor subfamily 4 group A member $2(N R 4 A 2))$. The clustering heatmap suggested that the samples could be obviously differentiated by the DEGs (Fig. 1).

\section{WGCNA analysis and enrichment analysis}

In order to meet the prerequisite of scale-free network distribution, the adjacency matrix weighting parameter "power" was explored. As a result, a "power" value of 19 was selected when the square of correlation coefficient first reached 0.8 (Fig. 2).

A co-expression network was constructed based on this "power" value. Firstly, the dissimilarity coefficients among the DEGs were calculated. Using the dissimilarity matrix, hierarchical clustering was performed to obtain a system clustering tree of the DEGs (Fig. 3A). According to the standards of a hybrid dynamic shear tree, the lowest gene number of each network module was set at 30. After network modules were identified using the dynamic shear method, the feature vector "eigengenes" was calculated for each module. Subsequently, the modules underwent clustering analysis, and the modules with close clustering relationships were merged into a new module. Finally, 


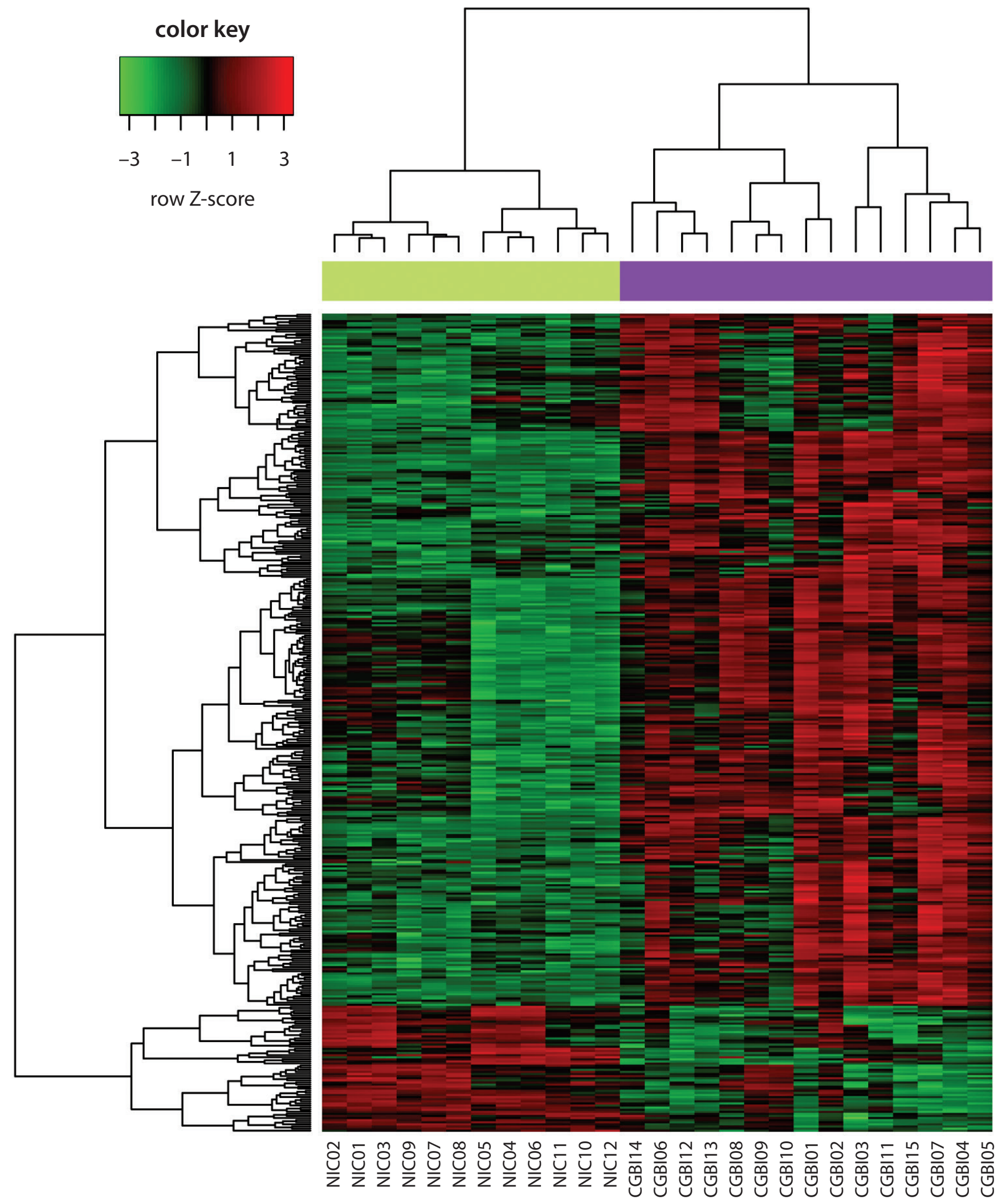

Fig. 1. Clustering heatmap for DEGs. Green and purple color in the sample strip separately represent the non-ischemic control (NIC) hippocampus samples and the complete global brain ischemia (CGBI) reperfusion hippocampus samples. The horizontal and vertical axes represent the clusters of samples and the clusters of genes, respectively

the DEGs were divided into 5 network modules (Fig. 3B), and the grey module was a set of the DEGs that could not be gathered into other modules.
To identify CGBI-associated modules, the feature vector of each module and CGBI was conducted with correlation analysis. The absolute values of correlation coefficients for 
A

scale independence

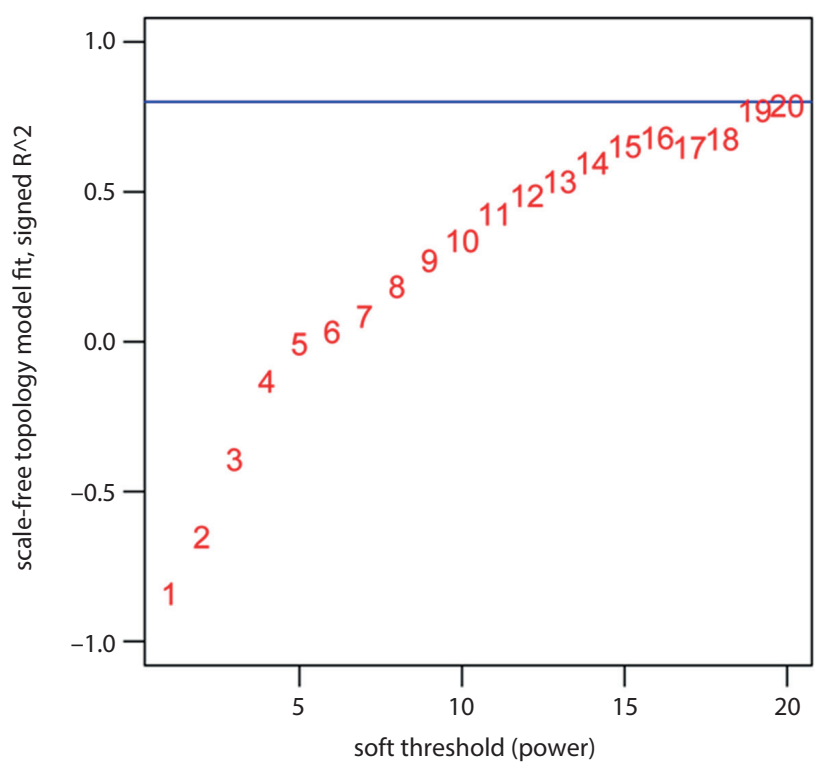

B

mean connectivity

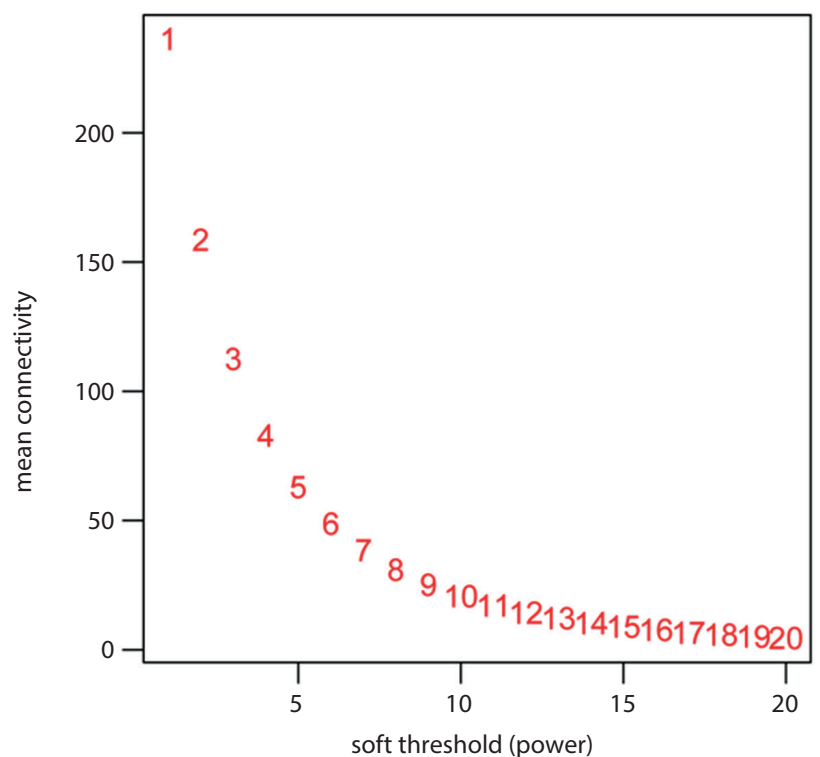

Fig. 2. The scale independence (A) and mean connectivity (B) for selecting the adjacency matrix weighting parameter "power". The higher the square of correlation coefficient, the closer the network is to scale-free network distribution. The blue line represents the standard line when the square of the correlation coefficient reaches 0.8

A

cluster dendrogram
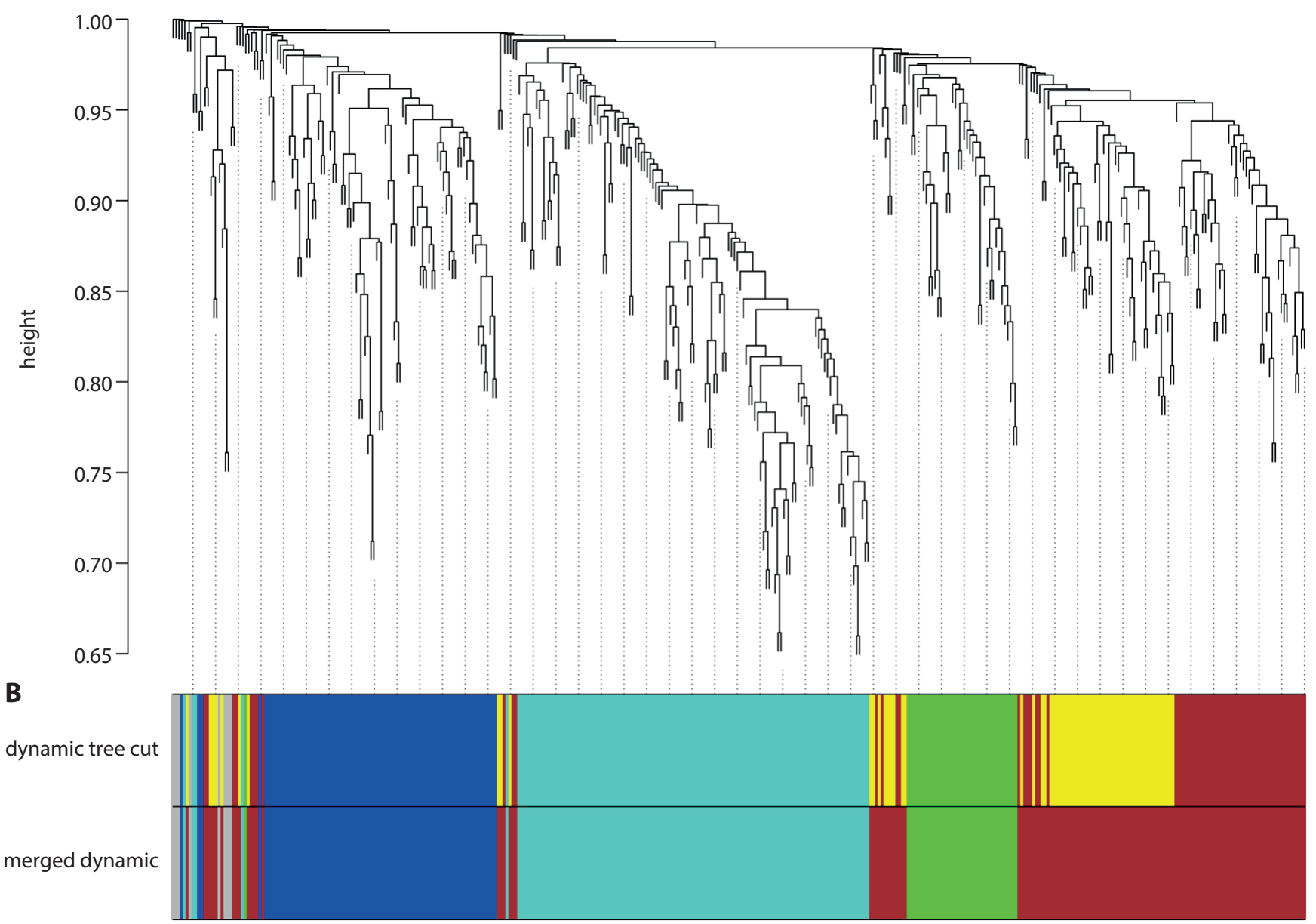

Fig. 3. The system clustering tree for the dissimilarity matrix (A), and the network modules before (dynamic tree cut) and after (merged dynamic) merging (B) 


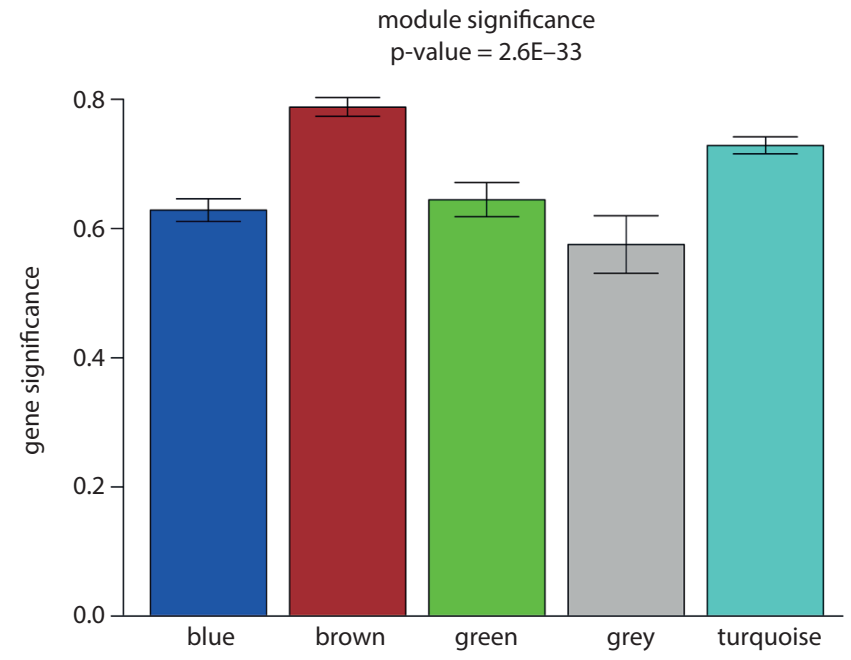

Fig. 4. The correlation graph of each module and complete global brain ischemia (CGBI). Horizontal axis and vertical axis separately represent module and gene significance

the modules were sorted, and it was found that the values for brown and turquoise modules were higher than 0.8 (Table 2). Meanwhile, the absolute values of gene significance for the modules were also calculated in order to screen CGBI-associated modules (Fig. 4). In addition, the heatmaps for the genes in the brown and turquoise modules are shown separately in Fig. 5A and 5B.

\section{Functional and pathway enrichment analysis}

The DEGs in the brown and turquoise modules were conducted with enrichment analysis. The DEGs in the brown module were mainly implicated in the positive regulation of gene expression (GO; p-value $=9.61 \mathrm{E}-09)$ and protein processing in the endoplasmic reticulum (KEGG; p-value $=2.86 \mathrm{E}-07)$. Also, the DEGs in the turquoise module were mainly involved in protein folding (GO; $\mathrm{p}$-value $=3.33 \mathrm{E}-07)$ and aminoacyl-tRNA biosynthesis $($ KEGG; p-value $=4.58 \mathrm{E}-07)($ Table 3$)$.

\section{PPI network analysis}

The DEGs in the brown and turquoise modules were merged, and a total of 259 DEGs were obtained (including 115 upregulated genes in the brown module, 115 upregulated genes in the turquoise module, 18 downregulated genes in the brown module, and 11 downregulated genes in the turquoise module). Next, the PPI network was built; it had 164 nodes and 620 edges (Fig. 6A). According to the degree values of the network nodes, heat shock protein 90 alpha family class A member 1 (HSP90AA1; degree $=47$ ) and heat shock protein 5 (HSPA5; degree $=25$ ) were key nodes. Additionally, HSP90AA1 and HSPA5 interacted in the PPI network.
Table 2 . The results of correlation analysis of each module and complete global brain ischemia

\begin{tabular}{|l|c|c|}
\hline \multicolumn{1}{|c|}{ Module } & Correlation coefficient & $p$-value \\
\hline Brown & 0.92 & $1.74 \mathrm{E}-11$ \\
\hline Turquoise & 0.83 & $1.06 \mathrm{E}-07$ \\
\hline Blue & 0.73 & $1.64 \mathrm{E}-05$ \\
\hline Green & 0.72 & $2.57 \mathrm{E}-05$ \\
\hline Grey & -0.75 & $5.85 \mathrm{E}-06$ \\
\hline
\end{tabular}

\section{TF-miRNA-target regulatory network analysis}

A total of 228 TF-miRNA-target regulatory relationships were obtained, involving 6 TFs, 10 miRNAs, and 99 targets (including 46 upregulated genes in the brown module, 45 upregulated genes in the turquoise module, 5 downregulated genes in the brown module, and 3 downregulated genes in the turquoise module). The TF-miRNA-target regulatory network is shown in Fig. 6B. In the regulatory network, the myelocytomatosis oncogene (MYC; TF, degree $=38$ ), heat shock transcription factor 1 (HSF1; TF, degree $=25)$, and $m i R-22($ degree $=12)$ had higher degree values. Importantly, MYC could target HSPA 5 in the regulatory network.

\section{qRT-PCR analysis}

The levels of HSPB1, HMOX1 and NR4A2 in the brain $\mathrm{I} / \mathrm{R}$ tissues and the control tissues were detected using qRT-PCR experiments. HSPB1 ( $<<0.001$; Fig. 7A) and HMOX1 ( $\mathrm{p}<0.001$; Fig. 7B) were significantly upregulated in the brain $\mathrm{I} / \mathrm{R}$ tissues compared with the control tissues, while NR4A2 (p < 0.001; Fig. 7C) was significantly downregulated. These findings were consistent with the results of differential expression analysis.

\section{Discussion}

In this study, 390 DEGs (including upregulated HSPB1 and $H M O X 1$, as well as downregulated NR4A2) were identified in the CGBI samples. Through WGCNA analysis, the brown and turquoise modules were screened as CGBIassociated modules. After the DEGs in the brown and turquoise modules were merged, a PPI network was built for them. In the PPI network, HSP90AA1 and HSPA5 were the key nodes. Moreover, MYC, HSF1 and miR-22 had higher degree values in the TF-miRNA-target regulatory network. Additionally, the qRT-PCR experiments confirmed upregulated $H S P B 1$ and $H M O X 1$ and downregulated NR4A2.

Oxidative stress can induce the phosphorylation of HSPB1 and HSPB5, which play neuroprotective roles 
Table 3. The Gene Ontology (GO) terms (A) and pathways (B) enriched for DEGs in the brown and turquoise modules

(A)

\begin{tabular}{|c|c|c|c|c|c|}
\hline Module & GO_ID & Biological process & Count & p-value & Genes \\
\hline \multirow{10}{*}{ Brown } & GO:0010628 & $\begin{array}{l}\text { positive regulation of gene } \\
\text { expression }\end{array}$ & 16 & $9.61 \mathrm{E}-09$ & $\begin{array}{c}\text { CCL3, RET, CAV1, SOX11, VIM, PARK2, HSPA1B, IL33, ATF4, ATF3, } \\
\text { VEGFA, BCL11A, NFE2L2, MSN, FGF2, PPP1R15A }\end{array}$ \\
\hline & GO:0030968 & $\begin{array}{l}\text { endoplasmic reticulum unfolded } \\
\text { protein response }\end{array}$ & 8 & $1.29 \mathrm{E}-08$ & HERPUD1, ATF3, STC2, YOD1, NFE2L2, PPP1R15A, EDEM1, DDIT3 \\
\hline & GO:0034976 & $\begin{array}{l}\text { response to endoplasmic reticulum } \\
\text { stress }\end{array}$ & 9 & 4.18E-08 & $\begin{array}{l}\text { ATF4, HERPUD1, STC2, TRIB3, HSPA5, PARK2, NFE2L2, PPP1R15A, } \\
\text { DDIT3 }\end{array}$ \\
\hline & GO:0006986 & response to unfolded protein & 5 & $1.62 \mathrm{E}-05$ & HERPUD1, CHAC1, PARK2, HSPA1B, DDIT3 \\
\hline & GO:0007568 & aging & 11 & 2.69E-05 & $\begin{array}{c}\text { CIQB, GSTA3, CRYAB, VIM, VEGFA, HSPB1, NFE2L2, PPPIR15A, } \\
\text { FGF2, DDIT3, TIMP1 }\end{array}$ \\
\hline & GO:0045944 & $\begin{array}{l}\text { positive regulation of transcription } \\
\text { from RNA polymerase II promoter }\end{array}$ & 19 & $5.74 \mathrm{E}-05$ & $\begin{array}{c}\text { CCL3, CAMTA2, SOX11, PTBP1, ARID5A, NR4A2, TEAD1, PARK2, IL33, } \\
\text { DDIT3, ATF4, ATF3, VEGFA, BCL111A, NFE2L2, USP16, NEUROD6, } \\
\text { FGF2, FOSL1 }\end{array}$ \\
\hline & GO:0043536 & $\begin{array}{l}\text { positive regulation of blood vessel } \\
\text { endothelial cell migration }\end{array}$ & 4 & $2.11 \mathrm{E}-04$ & VEGFA, HSPB1, NFE2L2, FGF2 \\
\hline & GO:0060548 & negative regulation of cell death & 6 & $2.20 \mathrm{E}-04$ & SOX11, PARK2, HSPA1B, NFE2L2, PPP1R15A, FGF2 \\
\hline & GO:0035690 & cellular response to drug & 6 & 3.46E-04 & MT2A, VEGFA, HSPA5, RNF149, NFE2L2, PPP1R15A \\
\hline & GO:0010629 & $\begin{array}{l}\text { negative regulation of gene } \\
\text { expression }\end{array}$ & 8 & $3.59 \mathrm{E}-04$ & CCL3, CDKN1A, STC2, CRYAB, SOX11, VEGFA, BCL11A, PARK2 \\
\hline \multirow{10}{*}{ Turquoise } & GO:0006457 & protein folding & 9 & 3.33E-07 & $\begin{array}{c}\text { ST13, TMX1, CCT5, HSP9OAA1, CCT4, AARS, DNAJA1, DNAJB4, } \\
\text { HSPA9 }\end{array}$ \\
\hline & GO:0051085 & $\begin{array}{l}\text { chaperone-mediated protein } \\
\text { folding requiring cofactor }\end{array}$ & 3 & 2.60E-03 & HSPH1, CCT4, HSPD1 \\
\hline & GO:0009409 & response to cold & 4 & 3.63E-03 & HSP9OAA1, HSPA2, RBM3, HSPD1 \\
\hline & GO:1902895 & $\begin{array}{l}\text { positive regulation of pri-miRNA } \\
\text { transcription from RNA polymerase } \\
\text { II promoter }\end{array}$ & 3 & $5.58 \mathrm{E}-03$ & JUN, STAT3, GNL3 \\
\hline & GO:0006366 & $\begin{array}{l}\text { transcription from RNA polymerase } \\
\text { II promoter }\end{array}$ & 8 & $9.52 \mathrm{E}-03$ & ARRB2, DBP, JUN, TARDBP, CEBPZ, DDX21, STAT3, GTF2H1 \\
\hline & GO:0009408 & response to heat & 4 & $1.12 \mathrm{E}-02$ & HSP90AA1, HSPA2, DNAJA1, HSPD1 \\
\hline & GO:0043066 & $\begin{array}{l}\text { negative regulation of apoptotic } \\
\text { process }\end{array}$ & 9 & $1.18 \mathrm{E}-02$ & CTH, JUN, NAA15, DNAJA1, MDM2, JAK2, HSPD1, NQO1, STAT3 \\
\hline & GO:1902728 & $\begin{array}{l}\text { positive regulation of growth factor } \\
\text { dependent skeletal muscle satellite } \\
\text { cell proliferation }\end{array}$ & 2 & $1.18 \mathrm{E}-02$ & JAK2, STAT3 \\
\hline & GO:1903748 & $\begin{array}{l}\text { negative regulation } \\
\text { of establishment of protein } \\
\text { localization to mitochondrion }\end{array}$ & 2 & $1.18 \mathrm{E}-02$ & HSPH1, DNAJA1 \\
\hline & GO:0045944 & $\begin{array}{l}\text { positive regulation of transcription } \\
\text { from RNA polymerase II promoter }\end{array}$ & 13 & $1.81 \mathrm{E}-02$ & $\begin{array}{c}\text { CCNL1, STAT3, PRPF6, GTF2H1, HSPH1, DBP, TARDBP, JUN, GTF2F1, } \\
\text { ETS2, CEBPZ, KDM3A, JAK2 }\end{array}$ \\
\hline
\end{tabular}

(B)

\begin{tabular}{|c|c|c|c|c|c|}
\hline Module & Pathway_ID & Pathway name & Count & $p$-value & Genes \\
\hline \multirow{3}{*}{ Brown } & rno04141 & $\begin{array}{l}\text { protein processing in endoplasmic } \\
\text { reticulum }\end{array}$ & 12 & $2.86 \mathrm{E}-07$ & $\begin{array}{c}\text { ATF4, HERPUD1, CRYAB, HSPA5, PARK2, DNAJB1, YOD1, HSPA1B, } \\
\text { NFE2L2, PPP1R15A, EDEM1, DDIT3 }\end{array}$ \\
\hline & rno04010 & MAPK signaling pathway & 11 & $1.08 \mathrm{E}-04$ & $\begin{array}{c}\text { DUSP5, ATF4, DUSP2, MAP2K3, GADD45G, HSPB1, HSPA1B, FLNC, } \\
\text { GADD45B, FGF2, DDIT3 }\end{array}$ \\
\hline & rno05205 & proteoglycans in cancer & 6 & $3.61 \mathrm{E}-02$ & CAV1, CDKN1A, VEGFA, MSN, FLNC, FGF2 \\
\hline \multirow{4}{*}{ Turquoise } & rno00970 & aminoacyl-tRNA biosynthesis & 8 & $4.58 \mathrm{E}-07$ & TARS, YARS, CARS, NARS, RARS, AARS, LARS, EPRS \\
\hline & rno03040 & spliceosome & 7 & $3.67 E-04$ & SRSF3, SF3B1, HSPA2, CWC15, PRPF6, PRPF $40 A$, RBM17 \\
\hline & rno03008 & ribosome biogenesis in eukaryotes & 5 & 3.36E-03 & NOP58, WDR43, RIOK2, GNL3, NMD3 \\
\hline & rno04915 & estrogen signaling pathway & 4 & 3.01E-02 & HSP9OAA1, HSPA2, FKBP5, JUN \\
\hline
\end{tabular}



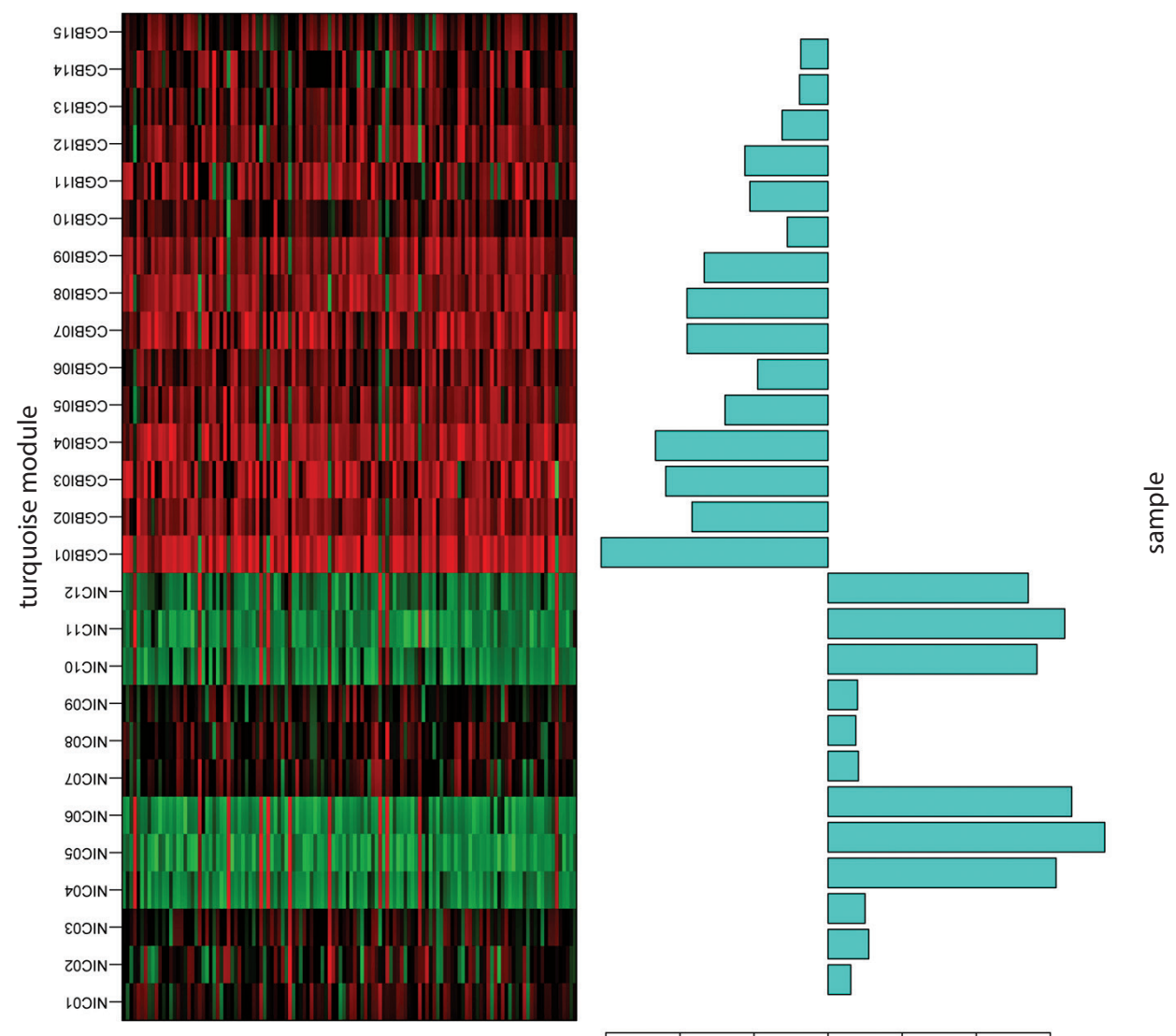

$\infty$
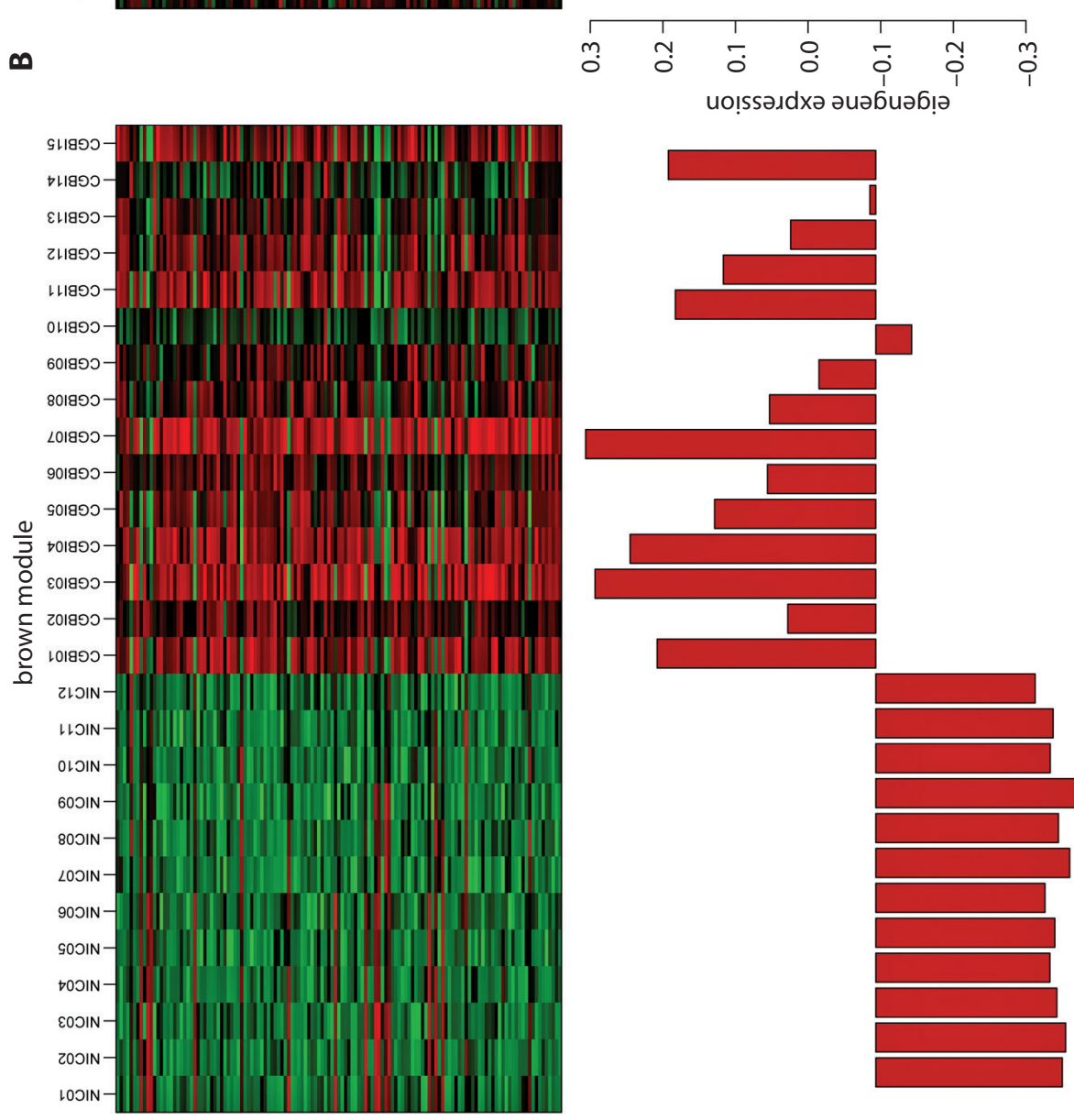

$\frac{0}{\frac{0}{0}}$

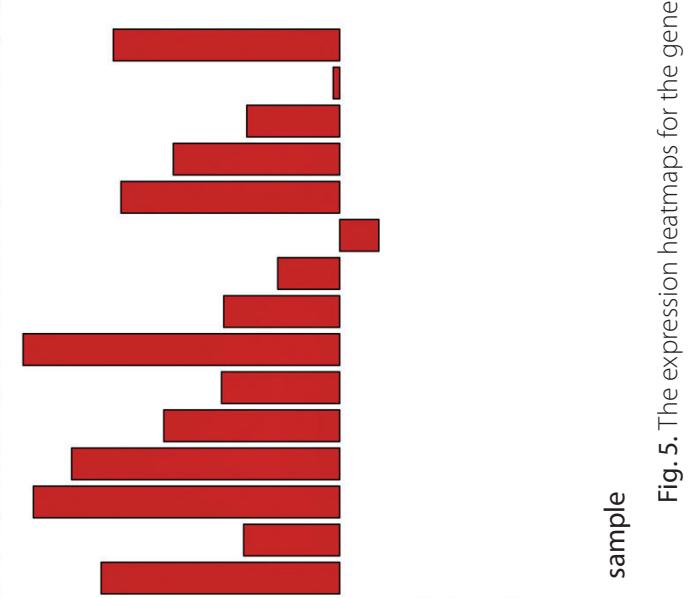

$\varangle$

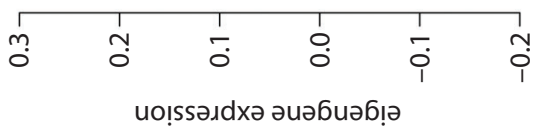



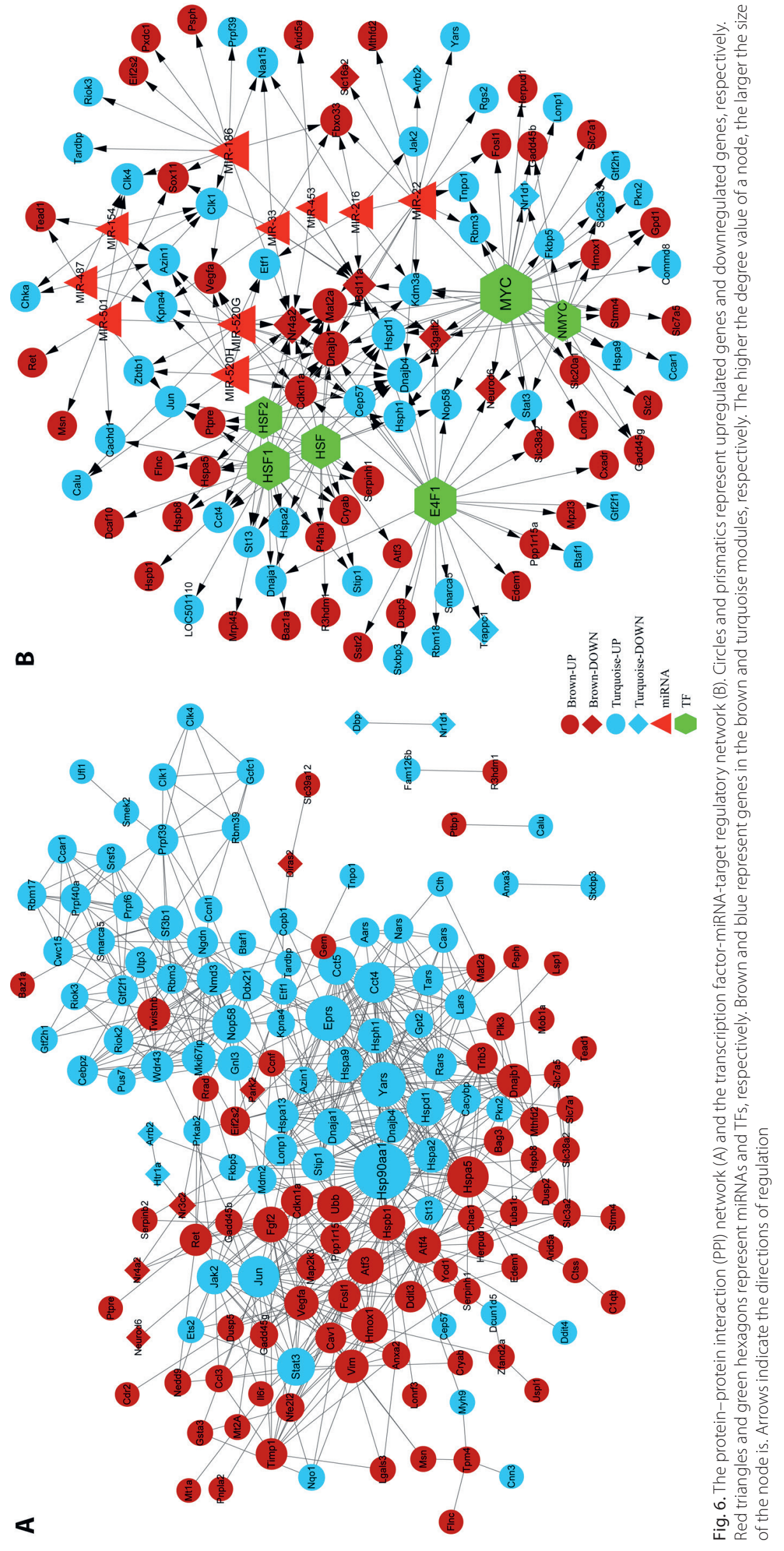

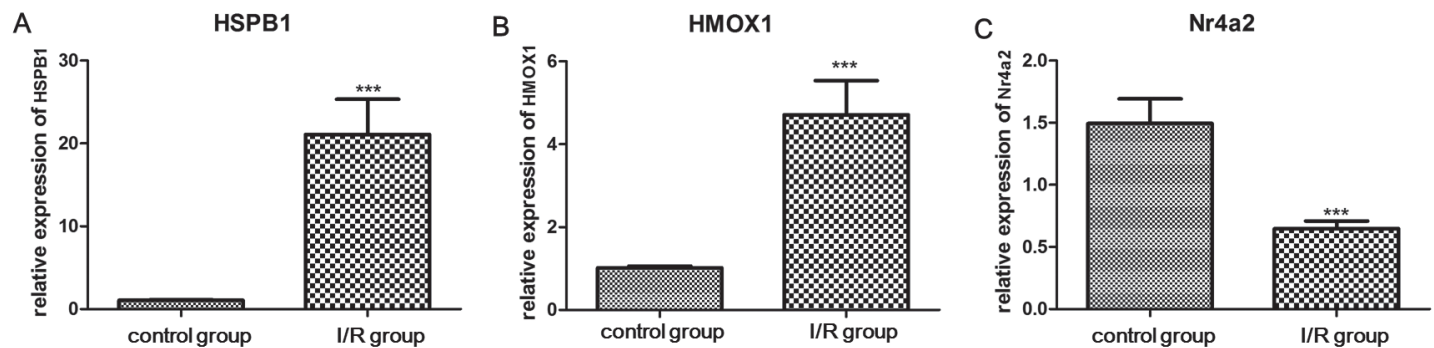

Fig. 7. The expression levels of HSPB1 (A), HMOX1 (B) and NR4A2 (C) in brain l/R tissues and control tissues

*** $p<0.001$

in hippocampal neurons. Kupffer cells, which are the main expression sites of hepatic $H M O X 1$, have anti-inflammatory effects and can resist the oxidative injury induced by I/R. ${ }^{26}$ NURR1 (also named NR4A2) contributes to intestinal regeneration following $I / R$ injury by suppressing p21 expression, which may provide new approaches for the therapy of intestinal I/R injury. ${ }^{27}$ These findings support the thesis that $H S P B 1, H M O X 1$ and $N R 4 A 2$ are related to the development and progression of I/R injury.

The mRNA expression of HSP90AA1 is reduced following $\mathrm{I} / \mathrm{R}$ and may be promoted by $m i R-1$ inhibition during myocardial I/R.28,29 A high protein expression of HSPA5 can exert neuroprotective effects and stop neural ischemic injury by attenuating endoplasmic reticulum (ER) stressinduced apoptosis. ${ }^{30}$ By negatively mediating ubiquitin carboxyl-terminal hydrolase isozyme L1 (UCHL1) and $H S P A 5$ protein levels, $m i R-181 b$ downregulation protects mice from ischemic injury and provides a therapeutic strategy for ischemic stroke. ${ }^{31}$ HSP90AA1 and HSPA5 interacted in the PPI network, suggesting that HSP90AA1 and HSPA5 might play roles in I/R injury through interaction with each other.

The MYC expression is upregulated after acute I/R injury and may promote the low expression of the anti-apoptotic $\mathrm{N}$-myc downstream-regulated gene 2 (NDRG2), which may be associated with myocardial apoptosis in I/R rats. ${ }^{32,33}$ By weakening $N F-\kappa B$ activation and reducing $M Y C$ expression, copper/zinc-superoxide dismutase (SOD1) overexpression helps to decrease ischemic damage. ${ }^{34}$ Granulocyte colony-stimulating factor (G-CSF) increases HSF1 expression by promoting phosphorylation and the interaction of the signal transducer and activator of transcription-3 (STAT3) with HSF1, which possesses cardio-protective effects in I/R mice. ${ }^{35} H S F 1$ prevents the death of cardiomyocytes following I/R partly by activating $A k t$ and inactivating caspase 3 and Jun $\mathrm{N}$-terminal kinase. ${ }^{36}$ These reports declared that $M Y C$ and $H S F 1$ might also be implicated in the mechanisms of I/R injury. MYC could target HSPA5 in the regulatory network, indicating a role of $M Y C$ in $\mathrm{I} / \mathrm{R}$ injury through mediation of HSPA5.

The $m i R-22$ plays a neuroprotective role by reducing inflammation and apoptosis, indicating that $m i R-22$ can be applied in the treatment of cerebral I/R injury. ${ }^{37}$ It can suppress the apoptosis of cardiomyocytes by targeting
CREB binding protein $(C B P)$; therefore, $m i R$-22 may serve as a novel target for preventing myocardial I/R injury. ${ }^{38,39}$ The $m i R-22$ inhibition helps to keep cardiac mitochondrial function, and thus has therapeutic potential for acute myocardial I/R injury. ${ }^{40} \mathrm{miR}-22$ decreases caveolin 3 (Cav3) expression and repairs endothelial nitric oxide synthase (eNOS) activity and NO production, inhibiting cardiac injury after I/R. ${ }^{41}$ Therefore, $m i R-22$ might be associated with the pathogenesis of $I / R$ injury by regulating the DEGs.

\section{Conclusions}

In conclusion, 390 DEGs were identified between CGBI and NIC samples. Also, HSPB1, HMOX1 and NR4A2 were the key genes associated with $\mathrm{I} / \mathrm{R}$ injury. Moreover, HSP90AA1, HSPA5, MYC, HSF1, and miR-22 might be implicated in the pathogenesis of I/R injury. However, the experimental study was insufficient and our results must still be further confirmed in subsequent studies.

\section{ORCID iDs}

Dingying Ma (1) https://orcid.org/0000-0001-7196-290X Jun Qiao (1) https://orcid.org/0000-0000-0000-0001 Qiang Qu (1) https://orcid.org/0000-0003-1786-887X Fei He (10) https://orcid.org/0000-0002-4157-0099 Wenhua Chen (1) https://orcid.org/0000-0003-4596-1764 Bo Yu (i) https://orcid.org/0000-0002-6652-9201

\section{References}

1. Breton A. Ischemia and reperfusion injury: When cells almost die. Vet Tech. 2010;31: E1-E6.

2. Dorweiler B, Pruefer D, Andrasi TB, et al. Ischemia-reperfusion injury: Pathophysiology and clinical implications. Eur J Trauma Emerg Surg. 2007;33(6):600-612.

3. Sirotković-Skerlev M, Plestina S, Bilić I, Kovac Z. Pathophysiology of ischaemia-reperfusion injury [in Croatian]. Lijec Vjesn. 2006;128 (3-4):87-95.

4. Kalogeris T, Baines CP, Krenz M, Korthuis RJ. Cell biology of ischemia/ reperfusion injury. Int Rev Cell Mol Biol. 2012;298:229-317.

5. de Groot H, Rauen U. Ischemia-reperfusion injury. Processes in pathogenetic networks: A review. Transplant Proc. 2007;39(2):481-484.

6. Gu JH, Ge JB, Li M, Wu F, Zhang W, Qin ZH. Inhibition of NF-kB activation is associated with anti-inflammatory and anti-apoptotic effects of ginkgolide $B$ in a mouse model of cerebral ischemia/reperfusion injury. Eur J Pharm Sci. 2012;47(4):652-660.

7. Qin XF, Lu XJ, Ge JB, Xu HZ, Qin HD, Xu F. Ginkgolide B prevents cathepsin-mediated cell death following cerebral ischemia/reperfusion injury. Neuroreport. 2014;25(4):267-273. 
8. Xi HJ, Zhang TH, Tao T, et al. Propofol improved neurobehavioral outcome of cerebral ischemia-reperfusion rats by regulating $\mathrm{BCl}-2$ and Bax expression. Brain Res. 2011;1410:24-32.

9. Li M, Zhang X, Cui L, et al. The neuroprotection of oxymatrine in cerebra ischemia/reperfusion is related to nuclear factor erythroid 2-related factor 2 (nrf2)-mediated antioxidant response: Role of nrf2 and hemeoxygenase-1 expression. Biol Pharm Bull. 2011;34(5):595-601.

10. Huang W, Liu X, Cao J, et al. miR-134 regulates ischemia/reperfusion injury-induced neuronal cell death by regulating CREB signaling. J Mol Neurosci. 2015;55(4):821-829.

11. Wang $H$, Tri AF, Chen X, Degracia DJ. Embryonic lethal abnormal vision proteins and adenine and uridine-rich element mRNAs after global cerebral ischemia and reperfusion in the rat. $J$ Cereb Blood Flow Metab. 2016;37(4):1494-1507.

12. Smith ML, Bendek G, Dahlgren N, Rosén I, Wieloch T, Bo KS. Models for studying long-term recovery following forebrain ischemia in the rat. 2. A 2-vessel occlusion model. Acta Neurol Scand. 1984;69(6):385-401.

13. Jamison JT, Kayali F, Rudolph J, Marshall M, Kimball SR, Degracia DJ. Persistent redistribution of poly-adenylated mRNAs correlates with translation arrest and cell death following global brain ischemia and reperfusion. Neuroscience. 2008;154(2):504-520.

14. Jamison JT, Szymanski JJ, Degracia DJ. Organelles do not co-localize with mRNA granules in post-ischemic neurons. Neuroscience. 2011; 199:394-400.

15. Szymanski JJ, Wang H, Jamison JT, Degracia DJ. HuR function and translational state analysis following global brain ischemia and reperfusion. Trans/ Stroke Res. 2013;4(6):589-603.

16. Carvalho BS, Irizarry RA. A framework for oligonucleotide microarray preprocessing. Bioinformatics. 2010;26(19):2363-2367.

17. Smyth GK. LIMMA: Linear models for microarray data. Bioinformatics and Computational Biology Solutions Using R and Bioconductor. 2011; 2011:397-420.

18. Liu X, Hu AX, Zhao JL, Chen FL. Identification of key gene modules in human osteosarcoma by co-expression analysis weighted gene co-expression network analysis (WGCNA). J Cell Biochem. 2017;118(11): 3953-3959.

19. Huang DW, Sherman BT, Lempicki RA. Systematic and integrative analysis of large gene lists using DAVID bioinformatics resources. Nat Protoc. 2009;4(1):44-57.

20. The Gene Ontology Consortium. Expansion of the gene ontology knowledgebase and resources. Nucleic Acids Res. 2017;45(Database issue):D331-D338.

21. Kanehisa M, Furumichi M, Tanabe M, Sato Y, Morishima K. KEGG: New perspectives on genomes, pathways, diseases and drugs. Nucleic Acids Res. 2017;45(D1):D353-D361.

22. Szklarczyk D, Morris JH, Cook H, et al. The STRING database in 2017: Quality-controlled protein-protein association networks, made broadly accessible. Nucleic Acids Res. 2017;45(Database issue):D362-D368.

23. Cline MS, Smoot M, Cerami E, et al. Integration of biological networks and gene expression data using Cytoscape. Nat Protoc. 2007;2(10): 2366-2382.

24. Wang J, Vasaikar S, Shi Z, Greer M, Zhang B. WebGestalt 2017: A more comprehensive, powerful, flexible and interactive gene set enrichment analysis toolkit. Nucleic Acids Res. 2017;45(W1):W130-W137.
25. Livak KJ, Schmittgen TD. Analysis of relative gene expression data using real-time quantitative PCR and the 2(-delta delta $C(T)$ ) method . Methods. 2001;25:402-408.

26. Devey L, Ferenbach D, Mohr E, et al. Tissue-resident macrophages protect the liver from ischemia reperfusion injury via a heme oxygenase-1-dependent mechanism. Mol Ther. 2009;17(1):65-72.

27. Zu G, Yao J, Ji A, et al. Nurr1 promotes intestinal regeneration after ischemia/reperfusion injury by inhibiting the expression of p21 (Waf1/Cip1). J Mol Med (Berl). 2016;95:1-13.

28. Guo W, Shan Z. GW26-e0722 repression of miR-1 is required for recovery of Hsp90aa1 against cardiac ischemia/reperfusion injury in rats. J Am Coll Cardiol. 2015;66(16 Suppl):C21.

29. Zuehlke AD, Beebe $K$, Neckers L, Prince T. Regulation and function of the human HSP90AA1 gene. Gene. 2015;570(1):8-16.

30. Peng W, Nan Z, Jia L, Jiefei L, Song H, Junfa L. Micro-RNA-30a regulates ischemia-induced cell death by targeting heat shock protein HSPA5 in primary cultured cortical neurons and mouse brain after stroke. J Neurosci Res. 2015;93(11):1756-1768.

31. Peng Z, Li J, Li Y, Yang X, Feng S, Han S. Downregulation of miR-181b in mouse brain following ischemic stroke induces neuroprotection against ischemic injury through targeting heat shock protein A5 and ubiquitin carboxyl-terminal hydrolase isozyme L1. J Neurosci Res. 2013;91(10):1349-1362.

32. Sun Z, Shen L, Sun X, et al. Variation of NDRG2 and c-Myc expression in rat heart during the acute stage of ischemia/reperfusion injury. Histochem Cell Biol. 2011;135(1):27-35.

33. Ma YL, Zhang LX, Liu GL, Fan Y, Peng Y, Hou WG. N-Myc downstreamregulated gene 2 (Ndrg2) is involved in ischemia-hypoxia-induced astrocyte apoptosis: A novel target for stroke therapy. Mol Neurobiol. 2017;54(5):3286-3299.

34. Huang $C Y$, Fujimura $M$, Noshita N, Chang YY, Chan PH. SOD1 downregulates NF-kappaB and c-Myc expression in mice after transient focal cerebral ischemia. J Cereb Blood Flow Metab. 2001;21(2):163-173.

35. Ma H, Gong H, Chen Z, et al. Association of Stat3 with HSF1 plays a critical role in G-CSF-induced cardio-protection against ischemia/ reperfusion injury. J Mol Cell Cardiol. 2012;52(6):1282-1290.

36. Zou Y, Zhu W, Sakamoto M, et al. Heat shock transcription factor 1 protects cardiomyocytes from ischemia/reperfusion injury. Circulation. 2003;108(24):3024-3030.

37. Yu H, Wu M, Zhao P, Huang Y, Wang W, Yin W. Neuroprotective effects of viral overexpression of microRNA-22 in rat and cell models of cerebral ischemia-reperfusion injury. J Cell Biochem. 2015;116(2):233-241.

38. Yang J, Chen L, Ding J, et al. MicroRNA-22 targeting CBP protects against myocardial ischemia-reperfusion injury through anti-apoptosis in rats. Mol Biol Rep. 2014;41(1):555-561.

39. Yang J, Yang J, Ding JW, Chen LH, Li S, Li XX. PM393 microRNA-22 protects against ischemia/reperfusion-induced myocardial injury via anti-apoptosis through its target gene Cbp. Global Heart. 2014;9:e141.

40. Du JK, Cong BH, Yu Q, et al. Upregulation of microRNA-22 contributes to myocardial ischemia-reperfusion injury by interfering with the mitochondrial function. Free Radic Biol Med. 2016;96:406-417.

41. Chen Z, Qi Y, Gao C. Cardiac myocyte-protective effect of microRNA-22 during ischemia and reperfusion through disrupting the caveolin-3/eNOS signaling. Int J Clin Exp Pathol. 2015;8(5):4614-4626. 